\title{
MOTION CLOSE TO THE HOPF BIFURCATION OF THE VERTICAL FAMILY OF PERIODIC ORBITS OF $L_{4}$
}

\author{
Mercè Ollé(1) Joan R. Pacha $^{(2)} \quad$ Jordi Villanueva ${ }^{(3)}$
}

31st October 2003

Departament de Matemàtica Aplicada I,

Universitat Politècnica de Catalunya

Diagonal 647, 08028 Barcelona, Spain.

(1) E-mail: merce.olle@upc.es

(2) E-mail: joanr@vilma.upc.es

(3) E-mail: jordi@vilma.upc.es

\begin{abstract}
The paper deals with different kinds of invariant motions (periodic orbits, $2 D$ and $3 D$ invariant tori and invariant manifolds of periodic orbits) in order to analyze the Hamiltonian direct Hopf bifurcation that takes place close to the Lyapunov vertical family of periodic orbits of the triangular equilibrium point $L_{4}$ in the $3 D$ restricted three-body problem (RTBP) for the mass parameter, $\mu$, greater than (and close to) $\mu_{R}$ (Routh's mass parameter). Consequences of such bifurcation, concerning the confinement of the motion close to the hyperbolic orbits and the $3 D$ nearby tori are also described.
\end{abstract}

Keywords: restricted problem, periodic orbits, Hopf bifurcation, invariant tori, invariant manifolds. 


\section{Contents}

1 Introduction 3

2 Linear stability of the vertical family of periodic orbits of $L_{4}$

$\begin{array}{lll}3 & \text { Rational collision } & 7\end{array}$

4 Irrational collision $\quad 9$

4.1 Parameterization and numerical computation of invariant curves . . . . . . 10

4.2 Hopf bifurcation of a family of invariant curves . . . . . . . . . . . . . . . 11

4.3 Linear normal behavior around the invariant curves . . . . . . . . . . . . 12

4.4 Dynamics in the neighborhood of the periodic orbits close to the transition 15

4.4.1 Invariant manifolds of the complex unstable periodic orbits . . . . . 15

4.4.2 Confinement and $3 D$ tori . . . . . . . . . . . . . . 17

$\begin{array}{ll}\text { References } & 19\end{array}$ 


\section{Introduction}

Our framework is the spatial circular restricted three body problem (RTBP from now on) as a Hamiltonian system of three degrees of freedom. This paper deals with the motion close to the so called vertical family of periodic orbits of the triangular points $L_{4}$ and $L_{5}$ of the RTBP.

Actually, there are several works devoted to the vertical family for values of the mass parameter $\mu$ with $\mu<\mu_{R}$ (the Routh critical value) - we mention [6], [8], [10] and [28] and references therein- but not as many for $\mu>\mu_{R}$ (see for instance [13]). Thus, in this work, we will concentrate on the case $\mu>\mu_{R}$ (but close to $\mu_{R}$ ), and we will study the motion related to the Hopf bifurcation of the vertical family which appears if we consider a mass parameter $\mu>\mu_{R}$ and we follow the family for big enough amplitudes.

This bifurcation takes place when a one parameter family of periodic orbits, of a Hamiltonian system with three degrees of freedom, undergoes a transition from stability to complex instability by means of a collision of characteristic multipliers in the unit circle. According to the value of the characteristic multipliers at the collision, we will distinguish between a the rational and irrational collision, and according to the effect of the nonlinear terms of the Hamiltonian, the bifurcation can be direct or inverse. In the case of the vertical family the bifurcation has always direct character, which means that stable objects (periodic orbits in the rational case and $2 D$ tori in the irrational one) bifurcate on the unstable side (around complex unstable periodic orbits).

In this paper, we will consider different kinds of "invariant motions" close to the critical periodic orbit in order to analyze the Hopf bifurcation (both rational and irrational cases). More precisely, we will consider periodic orbits, $2 D$ and $3 D$ invariant tori and the unstable and stable invariant manifolds of the hyperbolic periodic orbits. These objects will be used to show, near the bifurcation, the strong confinement of the solutions close to the invariant manifolds of the hyperbolic periodic orbits, due to the approximate coincident character of the stable and unstable manifolds.

Actually the Hopf bifurcation for a Hamiltonian system of three degrees of freedom has been analyzed (both numerically and analytically) by several authors, either considering the Hamiltonian itself or a one parameter family of $4 D$ symplectic mappings having a fixed point undergoing a Hopf bifurcation for a critical value of the parameter (for example, a suitable Poincaré map of the Hamiltonian using the energy as a parameter). More precisely, their analytical approach consists in considering the Hamiltonian (the mapping) around the transition periodic orbit (fixed point for the critical value) and reduce it to a normal form up to a finite order plus a remainder; afterwards, they analyze the dynamics from the truncated normal form (which turns out to be integrable).

In the context of symplectic mappings, we mention [4] for an analysis of the rational collision and [3] for the irrational one -from an analytical point of view-, and [12], [15] and [22] -from a numerical one-. Concerning the Hamiltonian itself and the irrational collision situation, we refer first to [9], where the normal form of the Hamiltonian, around the critical orbit, is obtained up to degree four. An analytical and complete treatment of this case has been recently carried out in [21] (see also [17] and [18]). In [21] a tricky algorithm to transform the Hamiltonian to normal form up to an arbitrary (finite) order 
is described, giving also quantitative estimates of the remainder (not in normal form) as function of the normalizing order and the distance to the critical orbit. The normal form is used to give an approximate description of the dynamics, and the bounds for the remainder are used to prove the existence of a cantor family of bifurcating $2 D$ tori (with big relative measure) when the whole (non integrable) Hamiltonian is considered. We also mention some papers dealing numerically with a Hamiltonian in the planetary mechanics context (see [16]) and in the galactic one (see [7], [20], [23] and [24]).

The aim of this paper is thus to show such bifurcation and the intricate dynamics related to it, for the vertical family of periodic orbits of the triangular points of the RTBP with $\mu>\mu_{R}$. This will be done from the numerical point of view, without any reduction to normal form. In this sense, let us point that a rigorous identification of the character of the bifurcation (direct or inverse) forces to do a nonlinear analysis of the normal form, but, from the numerical point of view, we will show the direct character by studying the stability of the bifurcated $2 D$ tori.

The contents of the paper are organized as follows. In Section 2 we introduce the vertical family of periodic orbits and discuss its linear character, showing it to be a natural candidate to have the transition stability-complex instability. Section 3 shows the typical behaviour of the bifurcation in the rational case. In section 4 we explore the irrational collision: on the one hand, the numerical computation and evolution of the $2 D$ invariant tori as well as the stable and unstable $2 D$ invariant manifolds of the complex unstable periodic orbit are described. On the other hand, the consequences of the bifurcation on the dynamics close to the periodic orbits before, at and after the transition are shown. In particular a strong confinement close to the hyperbolic periodic orbits and a change of topology on the nearby $3 D$ tori are also described.

\section{Linear stability of the vertical family of periodic orbits of $L_{4}$}

The circular spatial restricted three body problem (RTBP) describes the motion of a particle in a $3 D$ (position) space under the gravitational attraction of two bodies (called primaries); we assume that the particle has negligible effect on the motion of the primaries, and that they describe circular orbits around their common center of mass (the origin of coordinates). It is usual to describe this problem in a rotating (synodical) system of coordinates such that, in suitable units of distance, mass and time, the primaries have masses $\mu \in(0,1 / 2]$ and $1-\mu$, and are fixed at the points $(\mu-1,0,0)$ and $(\mu, 0,0)$. Denoting by $(x, y, z)$ the position of the massless particle in the synodical system, we define the corresponding momenta as $p_{x}=\dot{x}-y, p_{y}=\dot{y}+x$ and $p_{z}=\dot{z}$. Then, the equations of motion for the particle can be written as an autonomous Hamiltonian system of three degrees of freedom, whose Hamiltonian reads (see [27])

$$
H\left(x, y, z, p_{x}, p_{y}, p_{z}\right)=\frac{1}{2}\left(p_{x}^{2}+p_{y}^{2}+p_{z}^{2}\right)+y p_{x}-x p_{y}-\frac{1-\mu}{r_{1}}-\frac{\mu}{r_{2}}
$$


being $r_{1}$ and $r_{2}$ the distances between the particle and the big and small primaries respectively, that is, $r_{1}^{2}=(x-\mu)^{2}+y^{2}+z^{2}$ and $r_{2}^{2}=(x-\mu+1)^{2}+y^{2}+z^{2}$.

It is well known that the RTBP has five equilibrium points: $L_{1,2,3}$, the so called Eulerian or collinear points (which lie on the $x$ axis) and $L_{4,5}$, the Lagrangian or triangular points, each of which form an equilateral triangle with the primaries in the $(x, y)$ plane, that is, they are located at points $L_{4,5}=\left(\mu-\frac{1}{2}, \pm \frac{\sqrt{3}}{2}, 0, \mp \frac{\sqrt{3}}{2}, \mu-\frac{1}{2}, 0\right)$. As the motion around $L_{4}$ and $L_{5}$ is symmetric, we will concentrate on the point $L_{4}$.

The Jacobian matrix at the triangular points has the characteristic exponents

$$
\lambda_{1}=\mathrm{i}, \quad \lambda_{2,3}=\sqrt{-\frac{1}{2} \pm \frac{1}{2} \sqrt{1-27 \mu(1-\mu)}},
$$

and $\lambda_{j+3}=-\lambda_{j}, j=1,2,3$. The pair $\pm \mathrm{i}$ gives rise to vertical oscillations with angular frequency equal to 1 ; and we also recall that the characteristic exponents are purely imaginary and different for $0<\mu<\mu_{R}=\frac{1}{2}(1-\sqrt{23 / 27}) \approx 0.03852$ (Routh's mass parameter) and $L_{4}$ is linearly stable. For $\mu=\mu_{R}$ the planar frequencies collide on the imaginary axis; this produces a change in the linear stability and for $\mu_{R}<\mu \leq 1 / 2, L_{4}$ becomes complex-unstable.

On the other hand, for any value of the mass parameter $0<\mu \leq 1 / 2$, the linear vertical oscillations associated with the pair $\pm \mathrm{i}$ become a family of periodic orbits of the RTBP (due to Lyapunov's center theorem, see [25]): the so called vertical family of $L_{4}$. This family can be locally parametrized by its vertical amplitude or by the value of $\dot{z}$ when the orbit cuts the hyperplane $z=0$ in positive sense.

Along the paper, we shall use extensively the following Poincaré section:

$$
\Sigma=\left\{\left(x, y, z, p_{x}, p_{y}, p_{z}\right) \in \mathbb{R}^{6} \mid z=0\right\} .
$$

Furthermore, the family can be parametrized by means of the energy level of its orbits (defined by the value of the Hamiltonian $H$ ), which turns out to be an increasing function of the vertical amplitude. Thus, if we consider $\Sigma_{h}$ the $4 D$ manifold defined by $\Sigma_{h}=$ $\Sigma \cap\{H=h\}$, then there is (locally) only one vertical orbit crossing $\Sigma_{h}$ for $h>h_{L_{4}}$.

For numerical purposes, it will be convenient to work both in the flow context (using the canonical variables in $\mathbb{R}^{6}$ ) and in the map context (in the $4 D$ manifold $\Sigma_{h}$ ). For the latter, we first remark that given an initial point $p=\left(x_{0}, y_{0}, 0, p_{x_{0}}, p_{y_{0}}, p_{z_{0}}\right) \in \Sigma_{h}, p_{z_{0}}$ is obtained from $x_{0}, y_{0}, p_{x_{0}}, p_{y_{0}}$ and the value of $h$ fixed; so from now on we shall write -abusing notation- $p=\left(x_{0}, y_{0}, p_{x_{0}}, p_{y_{0}}\right)$ for those points $p$ on the surface of section $\Sigma_{h}$. Now we define the Poincaré map $P_{h}: \Sigma_{h} \rightarrow \Sigma_{h}$ by $P_{h}(p)=q$ where $q=\left(x, y, p_{x}, p_{y}\right) \in \Sigma_{h}$ such that the solution of the $3 D$ RTBP, starting at $p$, crosses the Poincaré section at the second passage. Since the flow comes from a Hamiltonian system, the map $P_{h}$ is symplectic. In particular, a family of periodic orbits, $2 D$ tori and $3 D$ tori in the flow context, becomes a family of fixed points, invariant curves and $2 D$ tori for the map.

The numerical continuation of the family can be done using the arc step method, which we briefly recall: a family of periodic points is regarded as a curve parametrized by the arc parameter $s$, that is,

$$
p(s)=\left(x_{0}(s), y_{0}(s), p_{x_{0}}(s), p_{y_{0}}(s)\right)
$$


such that

$$
G(s)=P_{h}(p(s))-p(s)=0 ;
$$

however, it is known that $p(s)$ also satisfies a suitable system of differential equations; therefore we may predict the successive points on the curve by a (low order) AdamsBashforth and we refine them using a modified Newton method (see [2], [8] and [26] for more details).

Concerning the stability of the periodic orbits of the vertical family, we note that if $\mu \neq \mu_{R}$, and at least for small vertical amplitudes, the linear stability of the periodic orbits of the family is the same as $L_{4}$. In particular, for a fixed $0<\mu<\mu_{R}$, the periodic orbits (for small amplitudes) of the vertical family are linearly stable, that is, for each periodic orbit, the corresponding four nontrivial - different from one - eigenvalues $(\lambda, 1 / \lambda$, $\sigma, 1 / \sigma)$ of the monodromy matrix lie on the unit circle; however, for $\mu>\mu_{R}$, the periodic orbits of the vertical family are (for small amplitudes) complex unstable, that is, the four eigenvalues leave the unit circle on a complex quadruple $(\lambda, \sigma=\bar{\lambda}, 1 / \lambda, 1 / \bar{\lambda}=1 / \sigma)$.

Nevertheless, the linear character may change for large enough amplitudes of the orbits, and, in fact, it does. In order to determine the stability or instability of the periodic orbits of a vertical family, we have computed - for each periodic orbit - the four nontrivial eigenvalues of the monodromy matrix, or equivalently, the stability parameters $\alpha$ and $\beta$ (see [5]) defined by the coefficients of the characteristic polynomial $p(z)$ of the monodromy matrix:

$$
p(z)=(z-1)^{2}\left(z^{4}+\alpha z^{3}+\beta z^{2}+\alpha z+1\right),
$$

and they satisfy

$$
\alpha=-(\lambda+1 / \lambda+\sigma+1 / \sigma), \quad \beta=2+(\lambda+1 / \lambda)(\sigma+1 / \sigma) .
$$

We plot in figure 1 the changes on the linear character of the orbits of the vertical families for any value of $\mu \in(0,0.5]$ and increasing values of $\dot{z}-\dot{z}=0$ corresponds to the equilibrium point $L_{4}-$. (see also [13]). In this figure we remark that, for a fixed value of $\mu>\mu_{R}$ (and close to $\mu_{R}$ ), the corresponding vertical family of periodic orbits has a transition from stability to complex instability (when decreasing $\dot{z}$ ), therefore there will be a critical (transition, resonant) orbit for which the four eigenvalues will collide in pairs on the unit circle (that is, $\lambda=1 / \bar{\lambda}=\exp (\mathrm{i} 2 \pi \kappa)$ ). We will show in the next sections that this collision gives rise to a direct Hopf-like bifurcation pattern and we will distinguish between the rational and irrational character of the collision. More precisely, we can choose suitable values of the mass parameter in order to obtain from figure 1 critical orbits with a rational or irrational collision of multipliers. According to the value of $\kappa$, rational $(\kappa=m / n)$ or irrational, then $n$-periodic orbits (that is, periodic orbits with a period close to $n$ times the period of the critical orbit) or $2 D$ invariant tori, respectively, may bifurcate on the unstable side (around the complex unstable periodic orbits). 


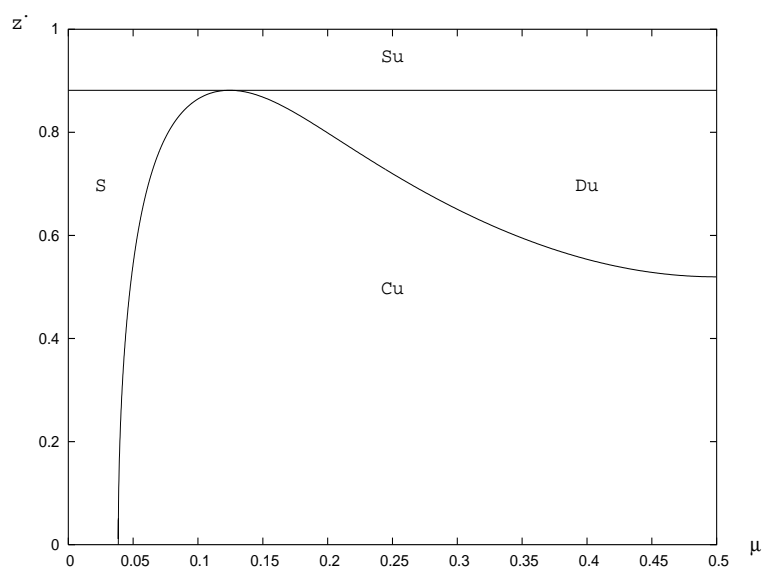

Figure 1: Change of the linear character of the orbits of the vertical family of $L_{4}$. We plot the mass parameter, $\mu$, on the horizontal axis and the positive vertical velocity, $\dot{z}$, when $z=0$ on the vertical one. S stands for stable (two couple of conjugate characteristic multipliers of modulus 1); $\mathrm{Cu}$ : complex-unstable (two conjugate eigenvalues outside the unit circle and their inverse ones); Du: double-unstable (two couple of positive eigenvalues); Su: semi-unstable (two conjugate eigenvalues of modulus 1 and a couple of positive eigenvalues).

\section{Rational collision}

In this section we want to discuss briefly the case of rational collision and the bifurcation of $n$-periodic orbits linked to it (see also [4] and [22]). In order to illustrate this phenomena, we select $\mu=0.069608 \ldots>\mu_{R}$ for which we have $\kappa=1 / 4$ at the transition orbit, that corresponds to a rational collision.

We have computed the vertical family of $L_{4}$ for this particular value of the mass parameter. Let us denote this family by $C$. Figure 2 (left) shows the path of the family $C$ on Broucke's diagram in the $(\alpha, \beta)$ plane (see also [5]). There the marked points $P_{1}, \ldots, P_{6}$ correspond to rational collisions $k=1 / n$ with $n=1, \ldots, 6$ from which families of $n$-periodic orbits might bifurcate. In this case, the critical orbit of the family $C$ corresponds to the point $P_{4}$. This $1 / 4$ rational collision produces two bifurcating families of 4-periodic orbits, that we will call $Q P_{1}$ and $Q P_{2}$. In figure 2 (right) we we consider the $(x, h)$ plane, being $x$ the initial condition (corresponding to $z=0$ and positive $\dot{z}$ ) and $h$ the value of the energy; we plot the evolution of the vertical family $C$ which starts at $L_{4}$ for $\mu=0.069608 \ldots$ and the branches $Q P_{1}$ and $Q P_{2}$ (which start at the critical orbit). The branch $Q P_{1}$ starts with periodic orbits that are stable and then become unstable, whereas all the periodic orbits of the branch $Q P_{2}$ are unstable (see also similar results for symplectic maps in [4] and $[22])$. For the details concerning the computation of the bifurcating branches of periodic orbits, we refer the interested reader to [2], [16], [23] and [26]. We have selected a member orbit of each family (the marked points in figure 2 right) and we also plot in figure 3 the $(x, y, z)$ projection of each orbit. Clearly, it can be seen that the bifurcating orbits have a period close to $4 T$ where $T$ is the period of the transition orbit. 

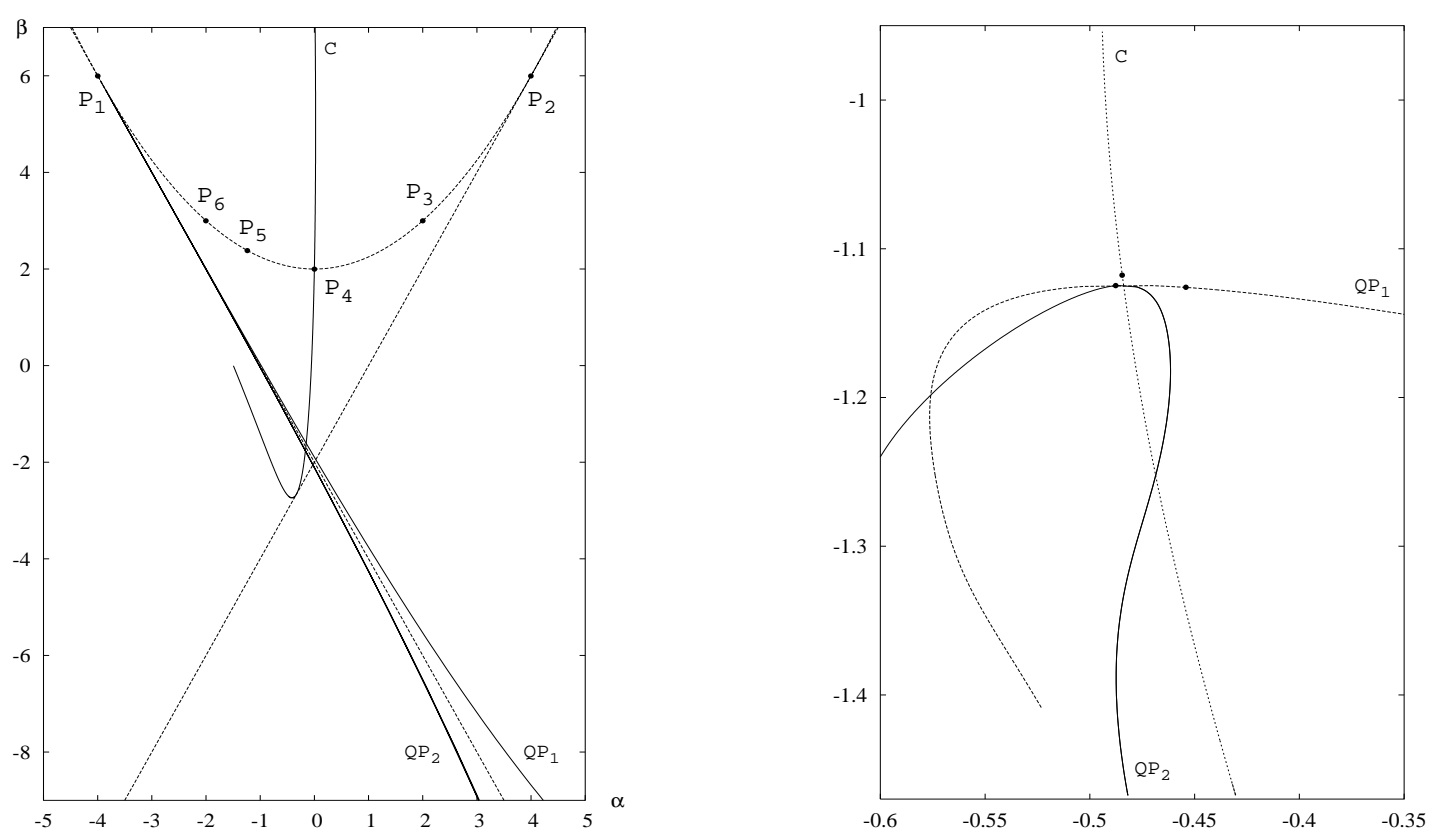

Figure 2: Left. Broucke's diagram. Stability of the vertical family $C$ for $\mu=0.069608 \ldots$ and of the bifurcated branches $Q P_{1}, Q P_{2}$ as well. Right. Again families $C, Q P_{1}$ and $Q P_{2}$; on the horizontal axis we plot the value $x$ of the initial condition (corresponding to $z=0$ and positive $\dot{z}$ ); and on the vertical one the value of the energy. A member (the marked point) of each family is also chosen (see figure 3 ).
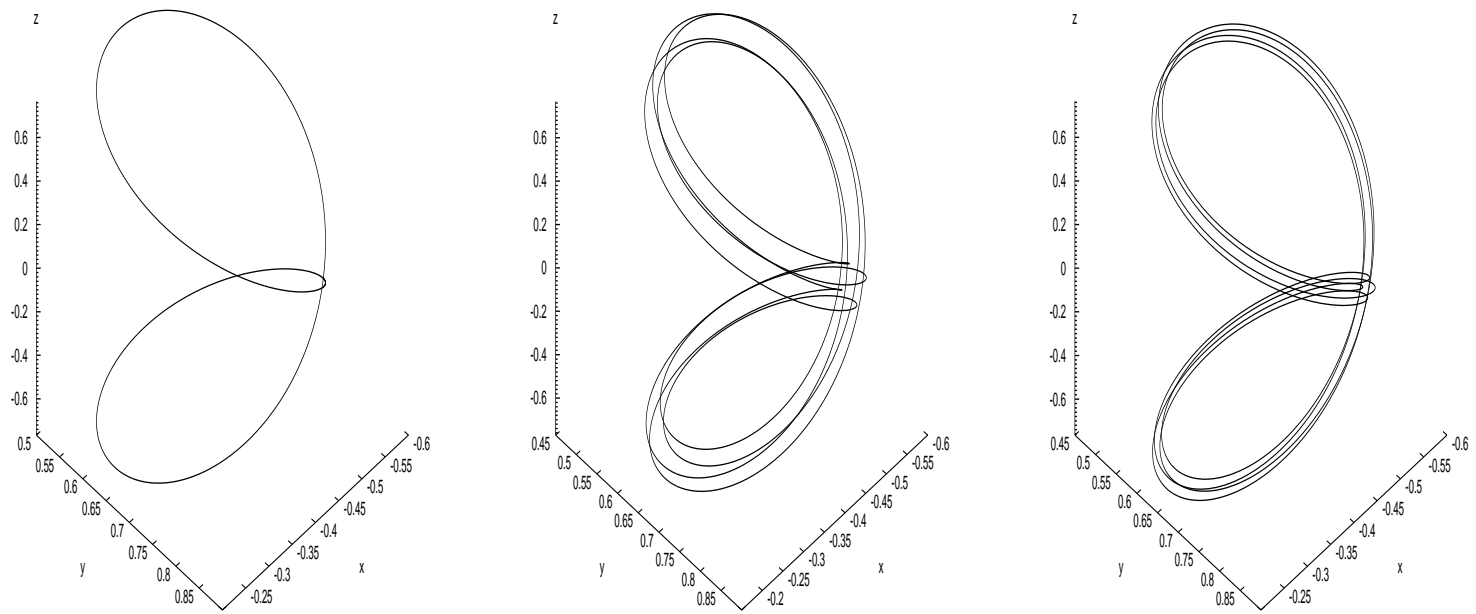

Figure 3: $(x, y, z)$ projection of a selected orbit of each family $C, Q P_{1}$ and $Q P_{2}$ respectively (see figure 2). Left: $h=-1.116234, T=6.335791$; middle: $h=-1.124786, T=$ 25.337930; right: $h=-1.1411396, T=25.309643$. 


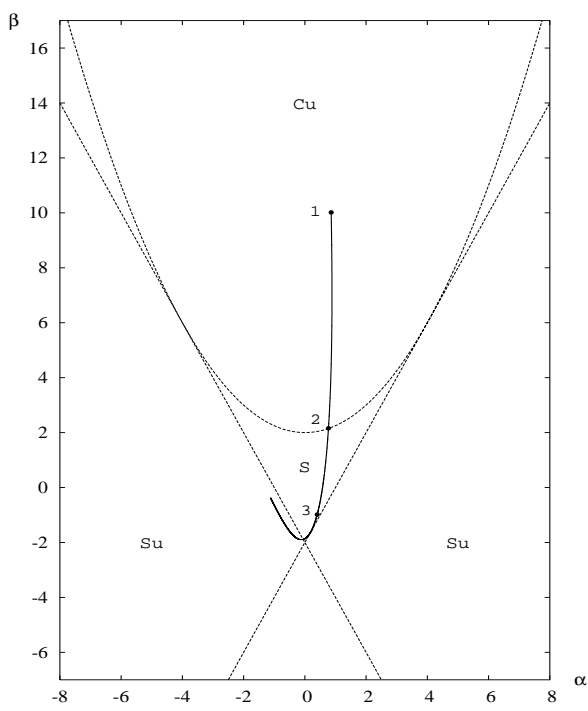

Figure 4: Broucke's diagram for the vertical family of $L_{4}$ for $\mu=0.04$.

\section{Irrational collision}

Let us now concentrate on the irrational collision. To illustrate the related behaviour, we take $\mu=0.04$, and we continue numerically the vertical family starting at $L_{4}$ (and increasing values of $\dot{z}$ when $z=0$, or equivalently increasing $h$ ). For each orbit in the family, we have computed also the associated stability parameters $\alpha, \beta$ and represented on Broucke's diagram (see figure 4). Therein, the transition (S-CU) orbit appears, now with an irrational $\kappa=0.291678572 \ldots$. We select two orbits (one complex-unstable and one stable, marked with dots 1 and 3 in figure 4$)$ and we plot their $(x, y, z)$ projection in figure 5 . We clearly see how the vertical amplitude increases as $\dot{z}$ grows.

Our aim in this section is to describe what happens around this transition. First, we summarize the results obtained: for $h>h_{\text {crit }}$, the vertical periodic orbit is (linearly) stable, so there are plenty of $3 D$ invariant tori around this orbit; there are also the two families of elliptic $2 D$ tori that are born at the periodic orbit - the so called Lyapunov families of $2 D$ tori - (see [12], [13] and [14]). For $h=h_{\text {crit }}$, both families become one family and it detaches, from the critical periodic orbit when $h<h_{\text {crit }}$, as a single family of elliptic $2 D$ invariant tori. Here, we remark that when $h$ decreases and crosses the critical value $h_{\text {crit }}$, the periodic orbit becomes hyperbolic: on the one hand, the elliptic $2 D$ tori unfold on the unstable side (at a finite distance of the periodic orbit) and, on the other hand, the $3 D$ unstable and stable invariant manifolds of each periodic orbit of the family become almost coincident (when $h$ is very close to and less than $h_{\text {crit }}$ ) and as a consequence, the motion is confined for a very long time in a small neighborhood of the unstable periodic orbit. The just described pattern of motion is known as the direct Hamiltonian-Hopf bifurcation in dimension three (see [3], [17], [21]), and it resembles the standard (direct) Hopf bifurcation in dimension two, where an elliptic periodic orbit detaches from the equilibrium point when this equilibrium point becomes unstable.

In the following subections we will show this behavior in detail. 

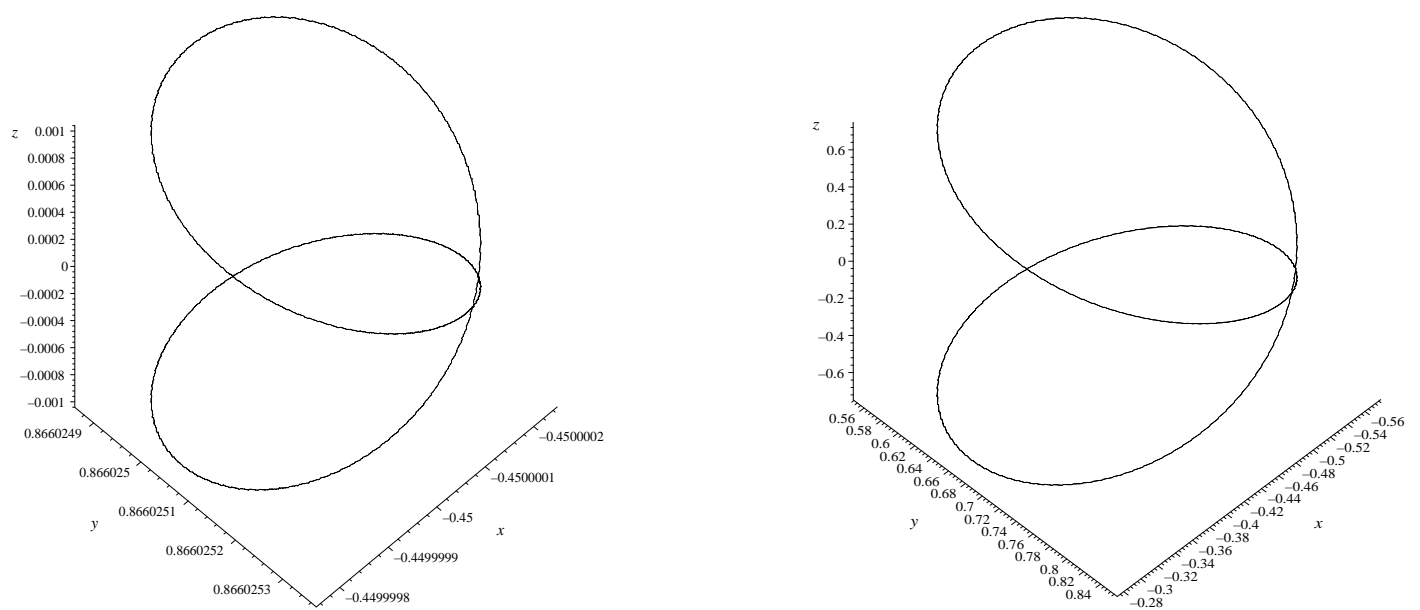

Figure 5: $(x, y, z)$ projection of a complex unstable orbit (left) and a stable one (right) that belong to the vertical family at $L_{4}$ for $\mu=0.04$ (see the marked points 1 and 3 on figure 4).

\subsection{Parameterization and numerical computation of invariant curves}

First, we want to study the behavior of $2 D$ tori (in the flow context), so we will compute the corresponding invariant curves for the Poincaré map (see definitions in section 2 ). We will assume, throughout the paper, that an invariant curve with frequency $\omega \notin 2 \pi \mathbb{Q}$ can be parameterized by $\theta \in \mathbb{T}^{1}=\mathbb{R} / 2 \pi \mathbb{Z} \mapsto X(\theta) \in \Sigma_{h}$ such that

$$
P_{h}(X(\theta))=X(\theta+\omega) .
$$

We remark that if $\left(\omega_{1}, \omega_{2}\right)$ is a suitable vector of frequencies of the 2-dimensional torus, for the flow, then $\omega=2 \pi \omega_{1} / \omega_{2}$ for the map. Equivalently, let $C\left(\mathbb{T}^{1}, \mathbb{R}^{4}\right)$ denote the space of continuous functions from $\mathbb{T}^{1}$ to $\mathbb{R}^{4}$; then $X(\theta)$ is a zero of the functional $F: C\left(\mathbb{T}^{1}, \mathbb{R}^{4}\right) \rightarrow$ $C\left(\mathbb{T}^{1}, \mathbb{R}^{4}\right)$ defined by

$$
F(X)(\theta)=P_{h}(X(\theta))-X(\theta+\omega) .
$$

The numerical method used to compute invariant curves has already been described in the flow context in [6] and for mappings in [11], [12] and [15]. We just recall that if we write $X(\theta)$ as a real Fourier series,

$$
X(\theta)=a_{0}+\sum_{k>0} a_{k} \cos (k \theta)+b_{k} \sin (k \theta), \quad k \in \mathbb{N}, \quad a_{k}, b_{k} \in \mathbb{R}^{4}
$$

then we should determine the Fourier coefficients such that they verify the equation $F(X)=0$. Actually we compute (numerically) a truncation of this series, that is

$$
X_{N}(\theta)=a_{0}+\sum_{k=1}^{N} a_{k} \cos (k \theta)+b_{k} \sin (k \theta), \quad N \in \mathbb{N},
$$


( $N \leq 50$ in our computations) and we determine the $2 N+1$ unknown coefficients $a_{0}, a_{k}$, $b_{k}\left(\right.$ in $\left.\mathbb{R}^{4}\right)$ as a solution of the discretized version of the equation $F(X)=0$ obtained when we impose that the equation $F\left(X_{N}\right)=0$ is satisfied only at the mesh of $2 N+1$ points on $\mathbb{T}^{1}: \theta_{j}=\frac{2 \pi j}{2 N+1}, 0 \leq j \leq 2 N$.

Let us remark finally that if $X(\theta)$ is a Fourier series corresponding to an invariant curve then, for any $\psi \in \mathbb{T}^{1}, Y(\theta) \equiv X(\theta+\psi)$ is a different Fourier series (i.e. with different Fourier coefficients) corresponding to the same invariant curve as $X(\theta)$. This introduces numerical difficulties when taking the linear system obtained and one applies the Newton method to solve the equation $F\left(X_{N}\right)=0$. In order to avoid this problem, we simply add an extra condition imposing that a component of $X(\theta)$ has a prescribed value when $\theta=0$. This will overcome the lack of uniqueness problem.

\subsection{Hopf bifurcation of a family of invariant curves}

It is well known that if the eigenvalues (also called characteristic multipliers) of the monodromy matrix associated with a periodic orbit are $\lambda_{i}$, for $i=1, \ldots, 6$, with $\lambda_{5}=\lambda_{6}=1$, then the eigenvalues of the differential of the Poincaré map, $D P_{h}$ at a fixed point are $\gamma_{i}=\lambda_{i}, i=1,2,3,4$. For $h>h_{c r i t}$, the periodic orbit of the vertical family is (linearly) stable, therefore, the eigenvalues of the matrix $D P_{h}$ at the corresponding fixed point are given by $\lambda_{1,2}=\exp \left( \pm \mathrm{i} \omega_{1}\right), \lambda_{3,4}=\exp \left( \pm \mathrm{i} \omega_{2}\right)$. The linear dynamics around this fixed point is given by the product of two harmonic oscillators, and it is proved that (under generic conditions) each linear oscillation gives rise to a Cantorian 1-parametric family of invariant curves with a frequency that tends to $\omega_{i}, i=1,2$, when the invariant curves tend to the fixed point (see [14]). We refer to these two families as Lyapunov families of invariant curves $-2 D$ tori for the flow itself - (see also [13], [12]).

For each invariant curve computed we consider its parameterization $X(\theta)$, its frequency $\omega$ and the initial point $X(0)=\left(\tilde{x}, \tilde{y}, \tilde{p}_{x}, \tilde{p}_{y}\right)$; for all the invariant curves we fix the value of $\tilde{x}$ (as explained above) and we take $\tilde{x}=-0.462$ (close to the value $x=\mu-\frac{1}{2}$ of the point $\left.L_{4}\right)$. In order to represent each invariant curve, we consider two coordinates $(\omega, \tilde{y})$; therefore, a family of invariant curves may be regarded as a curve in the plane $(\omega, \tilde{y})$.

We compute several families of invariant curves to show the direct Hopf bifurcation pattern. Actually, these are Cantorian families of invariant curves, but the holes are too small to be detected with the standard double precision arithmetic of the computer; so, from the numerical point of view, we deal with these families as if they were continuous. Figure 6 shows the two Lyapunov families of invariant curves (in the plane $(\omega, \tilde{y})$ ) for a fixed $h=-1.457018>h_{\text {crit }}$ (the lowermost curves in the figure). For each family, we see how the frequency tends to $\omega_{i}, i=1,2$, that is, the two families of invariant curves tend to the same fixed point (which is the critical periodic orbit for the flow) that has two different characteristic multipliers $\left(\lambda_{1}=\exp \left(\mathrm{i} \omega_{1}\right)\right.$ and $\left.\lambda_{3}=\exp \left(\mathrm{i} \omega_{2}\right)\right)$.

As mentioned above, when $h$ decreases and tends to $h_{\text {crit }}=-1.45714146$, the two families get closer and become a single family for $h=h_{\text {crit }}$. For $h<h_{\text {crit }}$, this family detaches from the periodic orbit in the sense that the family of invariant curves exists at a finite (and different from zero) distance from the periodic orbit which now is complex unstable. We see the evolution of this single family in figure 6 for for $h=-1.458308<$ 


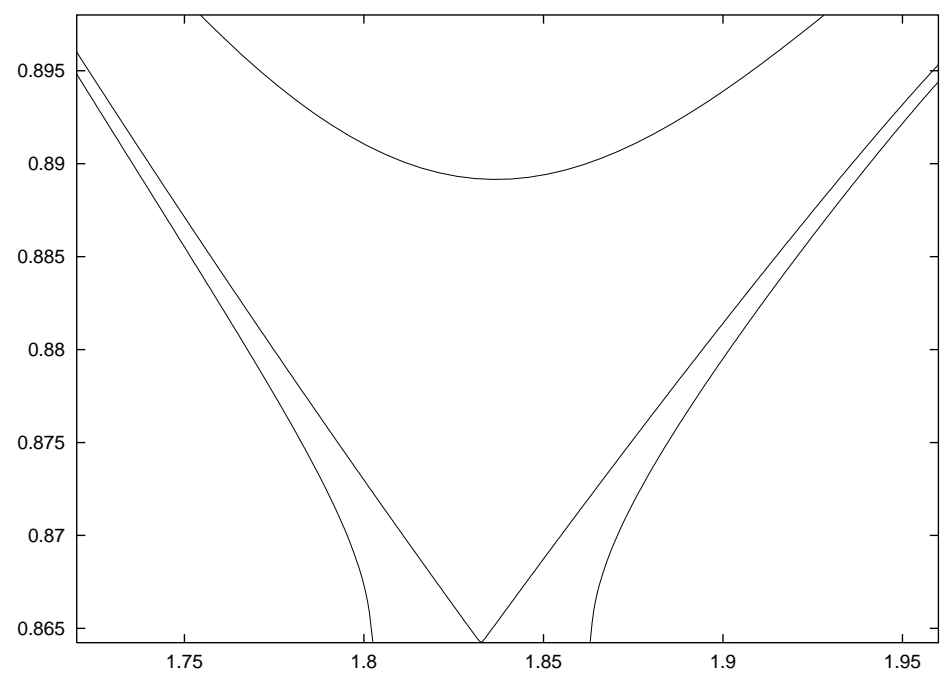

Figure 6: $\mu=0.04$. The two Lyapunov families of invariant curves (in the $(\omega, \tilde{y})$ plane) for $h=-1.457018>h_{\text {crit }}$; these are the two lowermost curves in the figure. We also plot the single family (for $h=h_{\text {crit }}$ ) and the detached family for $h=-1.458308<h_{\text {crit }}$.

$h_{\text {crit }}$; as h decreases the distance of the invariant curves to the periodic orbit increases.

We also show in figure 7 (left) an invariant curve of the Lyapunov family near the stable vertical periodic orbit $(h=-1.457018, \omega=1.93)$ and another one of the family on the unstable region for the same value of $\omega$ and $h=-1.458308$. If we follow the flow, the corresponding $2 D$ tori are plotted in figure 7 (right).

\subsection{Linear normal behavior around the invariant curves}

Now we assume that $\theta \in \mathbb{T}^{1} \rightarrow X(\theta) \in \Sigma_{h}$ is an invariant curve with frequency $\omega$ of the Poincaré map $P_{h}$. Then its linearized behavior is described by the 4-dimensional dynamical system

$$
\left.\begin{array}{rl}
\bar{X} & =A(\theta) X \\
\bar{\theta} & =\theta+\omega
\end{array}\right\}
$$

where $A(\theta)=D P_{h}(x(\theta))$. This kind of system is sometimes known as linear quasi-periodic skew-product.

The system (3) is called reducible if there exists a $2 \pi$ periodic (and maybe complex) change of variables $X=C(\theta) Y, \theta \in \mathbb{T}^{1}$, such that (3) becomes

$$
\left.\begin{array}{rl}
\bar{Y} & =B Y \\
\bar{\theta} & =\theta+\omega
\end{array}\right\}
$$

where the matrix $B \equiv C^{-1}(\theta+\omega) A(\theta) C(\theta)$ does not depend on $\theta$. Of course the dynamics of (4) can be easily described by computing the eigenvalues of $B$. As we do not know a direct method to compute the matrix $C(\theta)$, we will consider an alternative method to compute the spectrum of $B$. 

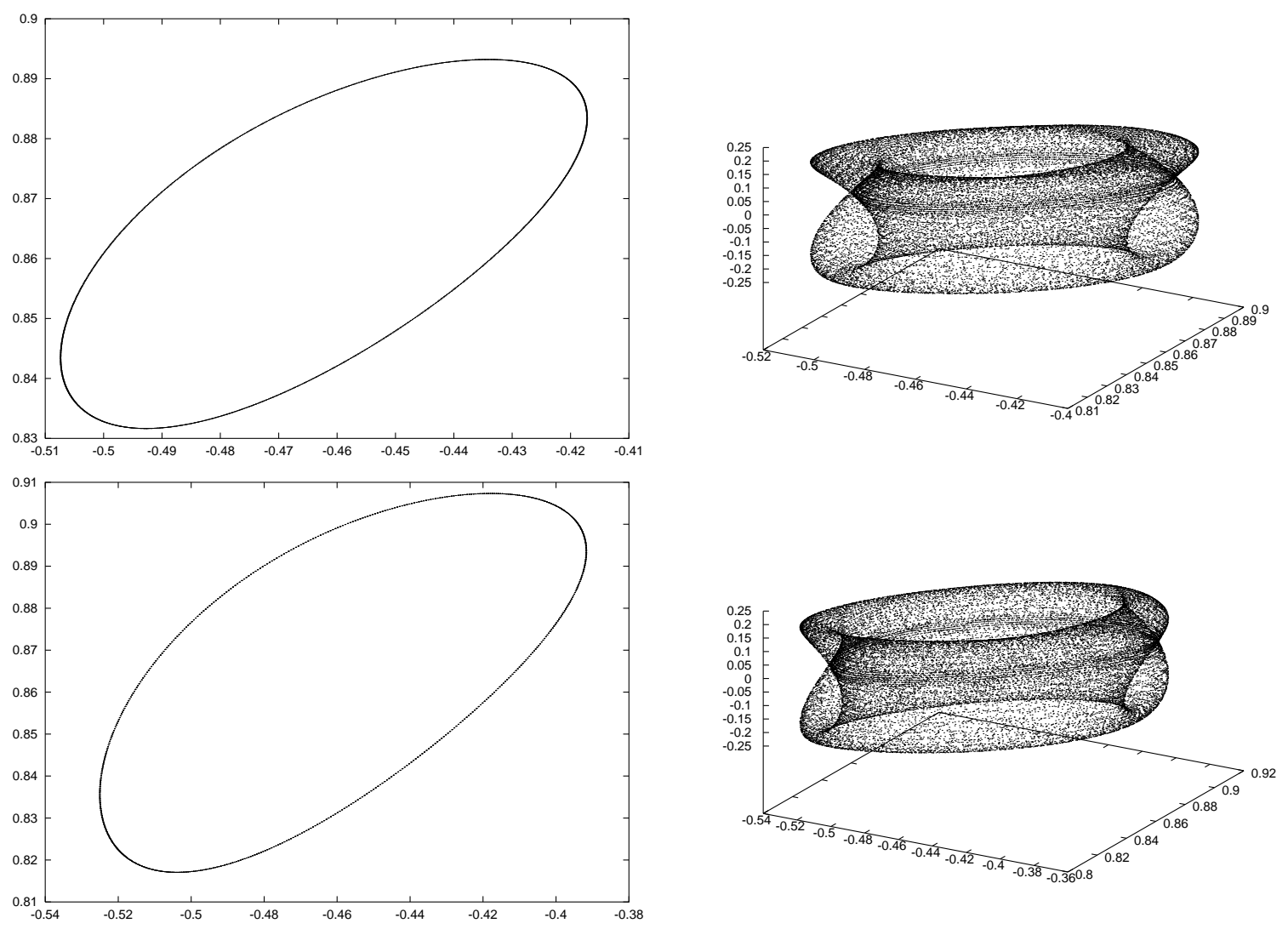

Figure 7: $\mu=0.04$. $2 D$ torus of the Lyapunov family of invariant tori on the stable region (top, with $\omega=1.90052495$ ) and on the complex unstable zone (bottom, with $\omega=1.79602495)$. Left: $(x, y)$ projection of the invariant curve in the Poincare section $z=0$. Right: $(x, y, z)$ projection of the corresponding torus under the flow.

Let us discuss the general $n$-dimensional case. Given $\psi \in C\left(\mathbb{T}^{1}, \mathbb{C}^{n}\right)$, we define the linear operator $L_{\omega}$ by

$$
\left(L_{\omega} \psi\right)(\theta)=A(\theta-\omega) \psi(\theta-\omega) .
$$

It turns out that the reducibility of (3) can be characterized in terms of the spectrum of $L_{\omega}$. That is, we first solve numerically the generalized eigenvalue problem

$$
\left(L_{\omega} \psi\right)(\theta)=\lambda \psi(\theta)
$$

and afterwards we take into account the following properties (the details and proofs can be found in [11]):

Proposition 1. Let $\lambda \in \mathbb{C}$ be an eigenvalue of $L_{\omega}$. Then, for any $k \in \mathbb{Z}, \lambda \exp (\mathrm{i} k \omega)$ is also an eigenvalue of $L_{\omega}$.

Definition. Two eigenvalues $\lambda_{1}$ and $\lambda_{2}$ of $L_{\omega}$ are said to be $\omega$-unrelated if $\lambda_{1} \neq \lambda_{2} \exp (\mathrm{i} k \omega)$ for all $k \in \mathbb{Z}$. 

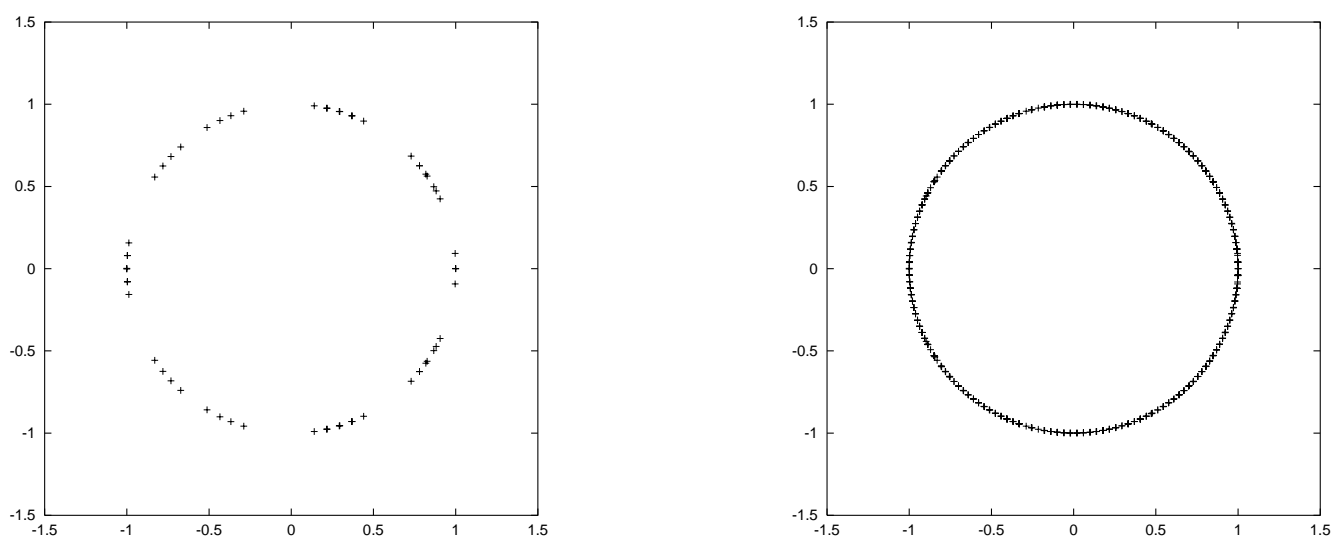

Figure 8: Eigenvalues of the discretized operator for $N=8$ (left) and $N=50$ (right).

Proposition 2. Assume that there exists $n \omega$-unrelated eigenvalues $\lambda_{1}, \ldots, \lambda_{n}$ for $L_{\omega}$. Then, (3) can be reduced to (4), where $B=\operatorname{diag}\left(\lambda_{1}, \ldots, \lambda_{n}\right)$.

From these results we conclude that an alternative way to compute the eigenvalues of $B$ is to approximate numerically the spectrum of $L_{\omega}$. We have followed this approach in this paper since we know that most of the invariant curves computed are reducible, that is, the corresponding linear dynamical system (3) is reducible (see [14] and [21]).

In particular, proposition 1 implies that the eigenvalues of the operator $L_{\omega}$ fill densely circles centered at the origin, and since we are in a Hamiltonian context, we remark that $B$ is a symplectic matrix therefore its eigenvalues are $1,1, \lambda, 1 / \lambda$. So an easy way to numerically determine the elliptic or hyperbolic character of an invariant curve is the following: we state that the invariant curve will be (linearly) stable if the eigenvalues of the operator $L_{\omega}$ lie on the unit circle of radius one, and unstable if the closure of the eigenvalues are three circles of radius one, $0<c<1$ and $1 / c$.

In order to implement numerically these results, we need a procedure to approximate the solutions of the generalized eigenvalue problem (6). The computations are based on the same discretization used in subsection 4.1 to compute invariant curves. This is, given a truncation value $N$, it is not difficult to derive the matrix (of dimension $4(2 N+1)$ ) which represents the truncation of $L_{\omega}$. Finally, the eigenvalues and eigenvectors of this matrix are computed by a standard numerical procedure. As an example we consider an invariant curve of the family with $h=-1.457018$, and we show in figure 8 the eigenvalues (in the complex plane) of the associated discretized operator $L_{\omega}$ for a truncation value $N=8$ (figure 8 left) and $N=50$ (figure 8 right). Since the eigenvalues lie on the unit circle, we conclude that this invariant curve is (linearly) stable.

It is also possible to derive heuristic estimates on the truncation error of the eigenfunctions from the decay of their Fourier coefficients and their size at the truncation point. Therefore, it is also possible to select, among the computed eigenfunctions, the most accurate ones -and, hence, the most accurate representants of the classes of $\omega$-related eigenvalues- for the actual truncation. We refer to [11] for a more complete description of these ideas and procedures.

This computation of the normal behavior has been carried out for all the Lyapunov 
families of invariant curves obtained and they turn out to be (linearly) stable (i.e. the eigenvalues of the corresponding matrix $B$ are $1,1, \lambda=\exp (\mathrm{i} \delta)$ and $1 / \lambda)$. Therefore we conclude that the Lyapunov families of (linearly) stable invariant curves persist on the unstable side (around the complex unstable periodic orbits) and they remain (linearly) stable. That is, we conclude that the irrational collision gives rise to the direct Hopf bifurcation pattern.

\subsection{Dynamics in the neighborhood of the periodic orbits close to the transition}

A natural question that arises is about the consequences -concerning the dynamics- of such direct Hopf bifurcation in the neighborhood of the family of periodic orbits close to the transition. In summary, we mention the following two features: the existence of the stable and unstable manifolds of the complex unstable periodic orbits produce a powerful confinement on the motion close to such periodic orbits. At the same time, the bifurcating elliptic $2 D$ tori on the hyperbolic side give rise to secondary tori ( $3 D$ tori close to the periodic orbits -after the transition to complex instability-) with a different topology from the $3 D \mathrm{KAM}$ tori. We describe the details below.

\subsubsection{Invariant manifolds of the complex unstable periodic orbits}

Let us show the behavior of the invariant manifolds -which are 3-dimensional- of the complex unstable periodic orbits. In order to approximate them numerically we regard again a periodic orbit as a fixed point of the Poincaré map $P_{h}$ and we compute the 2dimensional invariant manifolds (in the map context) of this fixed point. So, let us fix the value of $H=h<h_{\text {crit }}$ and let $p=\left(x_{0}, y_{0}, p_{x_{0}}, p_{y_{0}}\right) \in \Sigma_{h}$ be the fixed point associated with the complex unstable periodic orbit. We compute the eigenvalues of the differential of the Poincaré map, $D P_{h}$, at the fixed point $p$; they are, $\lambda_{1,2}=r_{1,2} \exp (\mathrm{i} \omega)$, with $0<r_{1}<1$, $r_{2}>1$ and $\lambda_{3,4}=r_{1,2} \exp (-\mathrm{i} \omega), \omega \in \mathbb{R}$. In order to approximate the unstable manifold of the fixed point $p$, we first select the eigenvalue $\lambda_{2}$ and we denote by $u+i v(u$ and $v$ are unitary vectors in $\mathbb{R}^{4}$ ) the associated eigenvector. As it is well known, the plane generated by $u$ and $v$ is a linear approximation to the $2 D$ real unstable manifold, therefore it is a good approximation to the manifold in a small neighborhood of the point. As we want to see how this manifold evolves also outside this small neighborhood, we consider the closed curve -called from now on the initial curve- on the linear approximation to the manifold given by $\left(x_{0}, y_{0}, p_{x_{0}}, p_{y_{0}}\right)+\sigma(s)$, with $\sigma(s)$ defined by

$$
\sigma(s)=c \frac{\tilde{\sigma}(s)}{\|\tilde{\sigma}(s)\|}, \quad \text { where } \quad \tilde{\sigma}(s)=\cos (s) u+\sin (s) v, \quad s \in[0,2 \pi]
$$

and $c$ is a small quantity. In order to see how the manifold behaves, we compute the iterates -through the map $P_{h^{-}}$of this curve (following the flow for fixed $h$ ).

We remark that the same computation can be done for the stable manifold, taking the eigenvalue $\lambda_{1}$, the corresponding eigenvector and following the flow through negative time; therefore using the map $P_{h}^{-1}$. 

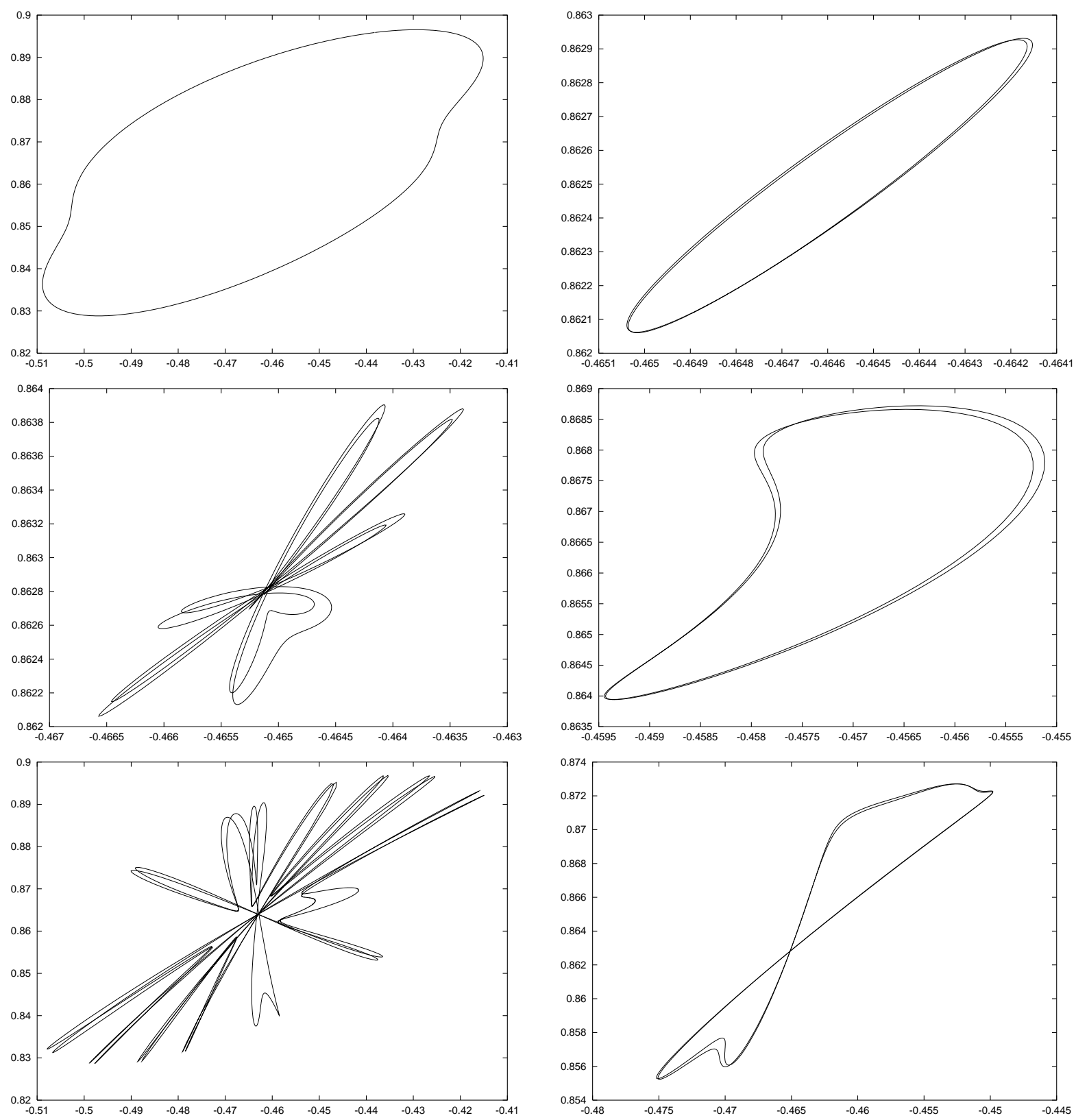

Figure 9: $\mu=0.04$. $(x, y)$ projection of the k-iterate, using $P_{h}$, of the initial curve $(7)$ on the unstable (left) and stable (right) manifolds of the complex periodic orbit with $h=-1.4577796221<h_{\text {crit }}$. Top: $k=100$, middle: $k=200$ and bottom: $k=300$.

As an example, we consider the hyperbolic periodic orbit with $h=-1.4577796221<$ $h_{\text {crit }}$ and the initial curve (7) on the unstable manifold. We compute the iterates (by means of $\left.P_{h}\right)$ of this initial curve and in figure 9 (left) we plot the k-th iterate $((x, y)$ projection) for $k=100,200$ and 300. These curves (all of them belonging to the unstable manifold) show the intricate to and fro behavior of the iterates on the invariant manifolds: the iterates go far away and then back (close) to the fixed point, repeatedly, in a chaotic 

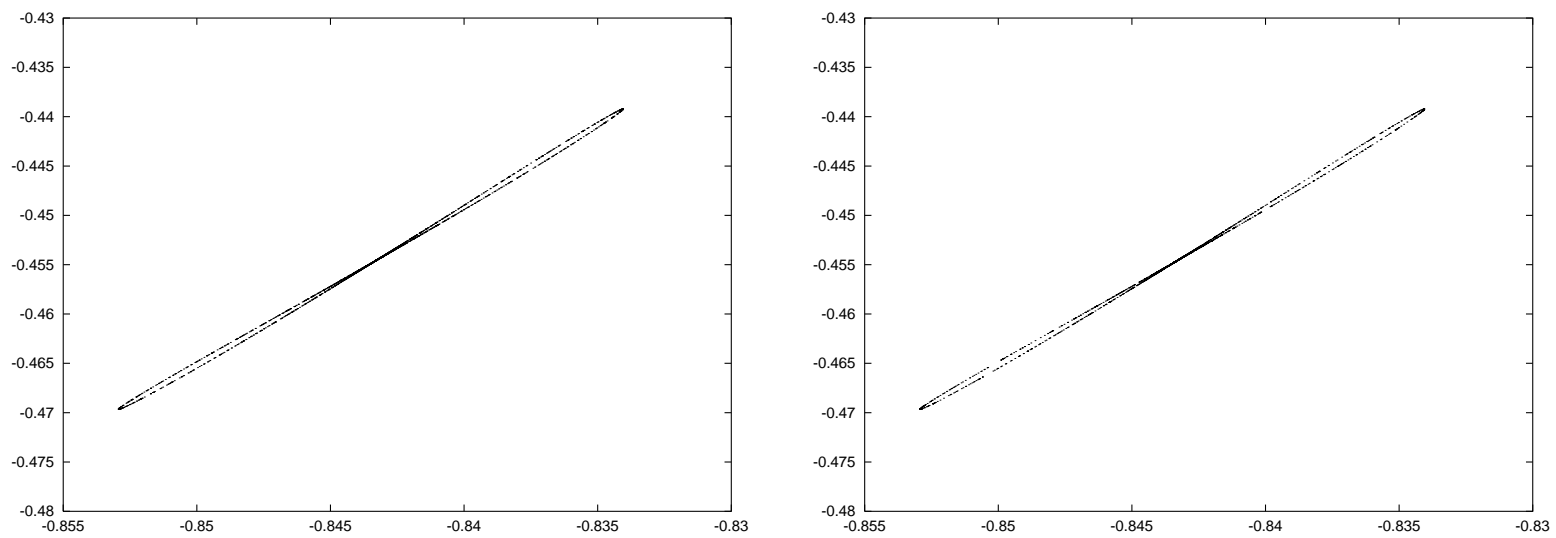

Figure 10: $\mu=0.04 .\left(p_{x}, p_{y}\right)$ projection of the slice $x=x_{0}$ (see the text) of the invariant unstable (left) and stable (right) manifolds of the complex unstable periodic orbit of figure 9 .

way. A similar computation is done for the stable manifold (see figure 9 right).

Another numerical approach to show that the invariant manifolds go far away and come back is by means of slices of the manifolds: given, as above, the fixed point $p=$ $\left(x_{0}, y_{0}, p_{x_{0}}, p_{y_{0}}\right) \in \Sigma_{h}$ (corresponding to the hyperbolic periodic orbit), we compute the slice of the invariant manifold defined by $x=x_{0}$. This slice turns out to be a curve and we plot (a piece of) it in figure 10 for the invariant unstable manifold (left) and for the stable one (right) of the complex unstable periodic orbit used in figure 9; apparently both slices coincide but they do not. This happens since we have only plotted the slice obtained when the unstable manifold leaves the fixed point and goes back near it for the first time. Actually, we know from the analytical results (see [18] and [21]), that if we consider the truncated and integrable normal form of the Hamiltonian around the critical orbit, then the unstable and stable invariant manifolds of a complex unstable periodic orbit coincide; however, when the complete (non integrable) Hamiltonian is regarded (that is, the truncated normal form plus the remainder), the splitting of separatrices appears and this produces the chaotic behavior of the iterates on the invariant manifolds observed in figure 9.

\subsubsection{Confinement and $3 D$ tori}

On the one hand, the almost coincidence of the manifolds somewhat trap the motion inside them giving rise to a powerful confinement in the sense that a point close to the unstable (or stable) manifold may follow the shape of the manifolds and remain confined near them for a long time (many iterates in the map context).

In order to show this behaviour, we consider the evolution of a $2 D$ KAM torus in $\Sigma_{h}$ ( $3 D$ torus in the flow context) before, at and after the transition. For $h=-1.44908826>$ $h_{c r i t}$, the fixed point (periodic orbit for flows) is stable so there are plenty of $2 D$ KAM tori; we plot in figure 11 (top left) the 5000 iterates -through $P_{h}$ - of an initial condition of a $2 D$ torus near the stable fixed point. In figure 11 (top right), we do the same computation for $h=h_{\text {crit }}=-1.45714146$; we remark now the bigger size in the plot window since for 

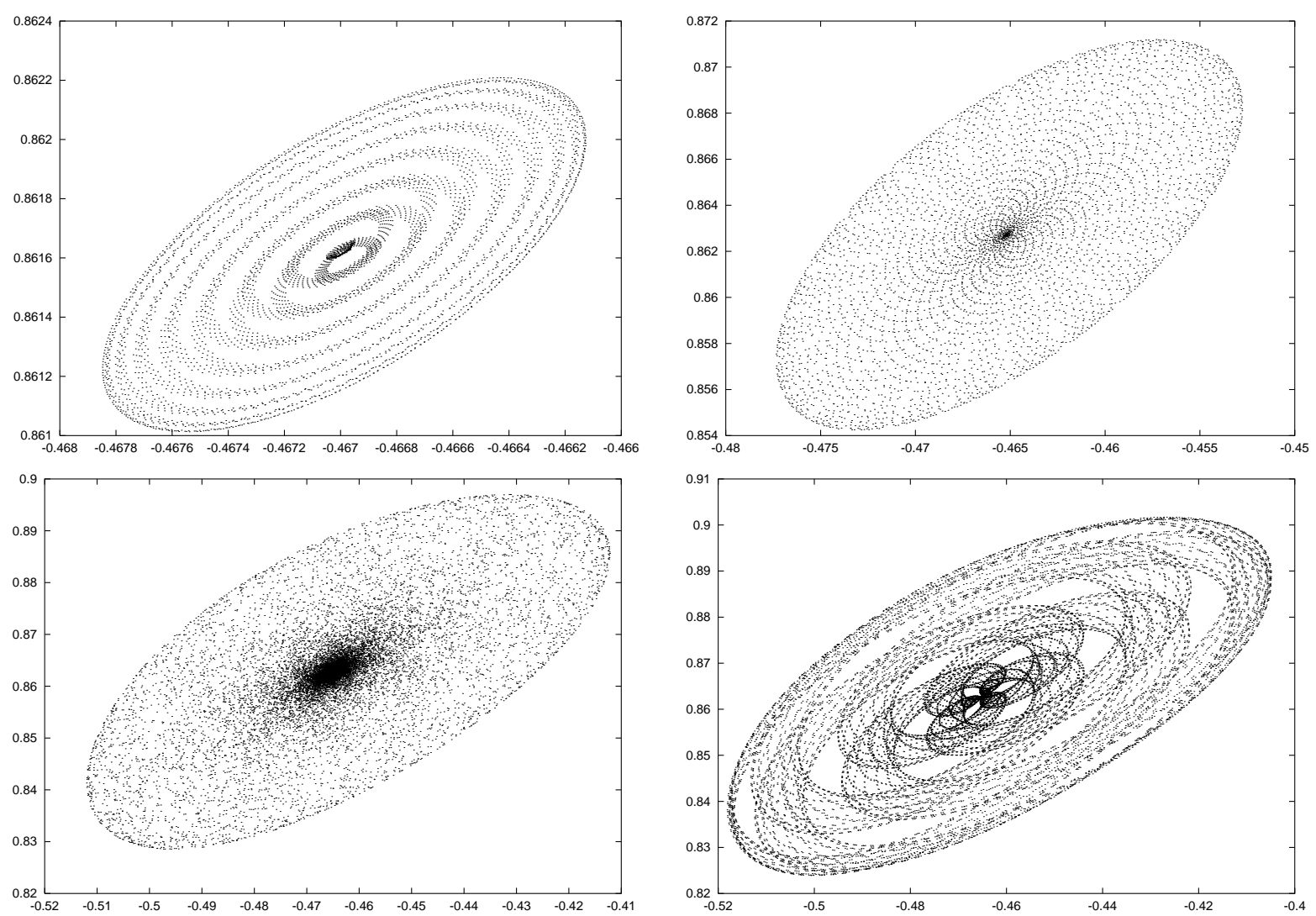

Figure 11: 5000 iterates $((x, y)$-projection) of initial conditions on a $2 D$ torus close to a stable (top left) and critical (top right) fixed point. 20000 iterates of the initial condition $p+\epsilon$ (see the text) close to a complex unstable periodic orbit; $\epsilon=10^{-6}$ (bottom left) and $\epsilon=10^{-4}$ (bottom right). We remark the different size for the frame in each case.

$h<h_{\text {crit }}$ the fixed point becomes complex unstable and its invariant manifolds play a key role. For $h=-1.45777962<h_{\text {crit }}$, we consider the corresponding hyperbolic fixed point $p=\left(x_{0}, y_{0}, p_{x_{0}}, p_{y_{0}}\right)$ and we take initial conditions $p=\left(x_{0}+\epsilon, y_{0}+\epsilon, p_{x_{0}}+\epsilon, p_{y_{0}}+\epsilon\right)$ (for $\epsilon>0$ small) close to it. We plot the 20000 iterates (using $P_{h}$ ) in figure 11 (bottom left with $\epsilon=10^{-6}$, and bottom right with $\epsilon=10^{-4}$ ). We remark again the even bigger size of both plots window since there do exist invariant manifolds in this case; we observe in figure 11 (bottom left) how the iterates follow the invariant manifolds shape and describe a chaotic orbit; actually the same picture is obtained if we plot the iterates of a point in the unstable manifold. On the other hand, for a bigger value of $\epsilon=10^{-4}$, the iterates in figure 11 (bottom right) seem to remain in a $2 D$ torus (either one $2 D$ KAM torus that survives the bifurcation or a secondary $2 D$ torus which is born from the existence of the stable bifurcating invariant curves); in any case, however, the $k$-iterates are confined for a large value of $k$.

We remark finally that, if the iterates are on a $2 D$ torus, they would be confined forever; but also Arnol'd diffusion (see [1]) may appear (see Nekhoroshev's type estimates of diffusion for a symplectic mapping in [19]). 
On the other hand, we also remark that the effect of the bifurcation is local in the following sense: concerning the $3 D \mathrm{KAM}$ tori (in the flow context) existing around a stable periodic orbit (before the transition), two different behaviors have been shown in figure 11: those tori close enough to the periodic orbit are sensitive to the bifurcation and they change the topology after the transition, since the $2 D$ bifurcating stable tori (after the transition) give rise to the secondary $3 D$ tori which have nothing to do with the KAM tori before the transition. Nevertheless, the 3D KAM tori far enough from the periodic orbit remain just after the transition as they are; however, as soon as the energy decreases, the invariant manifolds also increase and may reach the further $3 D$ tori anyway.

\section{Acknowledgements}

This work has been partially supported by the Catalan CIRIT grant number 2001SGR-70, the Spanish grant number BFM2000-0623 and the INTAS grant number 00-221.

\section{References}

[1] V. I. ARnol'D, Diffussion of dynamical systems with several degrees of freedom, Sov. Math. Dokl. 5:3 (1964), 581-585.

[2] E. Belbruno, J. Llibre, M. Ollé, On the families of periodic orbits which bifurcate from Sitnikov motions, Cel. Mech. Dynam. Astron. 60 (1994), 99-129.

[3] T. J. Bridges, R. H. Cushman, R. S. Mackay, Dynamics near an irrational collision of eigenvalues for symplectic mappings, Fields Institute Communications 4 (1995), 61-79.

[4] T. J. Bridges, J. E. Furter, Singularity theory and equivariant symplectic maps, Lecture Notes in Mathematics 1558 (1993), Springer-Verlag.

[5] R. Broucke, Stability of periodic orbits in the Elliptic Restricted Three-Body Problem, AIAA J. 7 (1969), 1003-1009.

[6] E. Castellà, A. Jorba, On the vertical families of two-dimensional tori near the triangular points of the bicircular problem, Cel. Mech. and Dynam. Astron. 76 (2000), $35-54$.

[7] G. Contopoulos, B. Barbanis, Periodic orbits and their bifurcations in a 3Dsystem, Cel. Mech. Dynam. Astron. 59 (1994), 279-300.

[8] G. Gómez, J. Llibre, R. Martínez, C. Simó, Dynamics and Mission Design Near Libration Points, World Scientific Monograph Series in Mathematics 2 (2001).

[9] D. G. HegGie, Bifurcation at complex instability, Cel. Mech. 35 (1985), 357-382. 
[10] A. JorBA, A numerical study on the existence of stable motions near the triangular points of the real Earth-Moon system, Astron. Astrophys. 364 (2000), 327-338

[11] A. JorbA, Numerical computation of the normal behaviour of invariant curves of n-dimensional maps, Nonlinearity 14 (2001), 943-976.

[12] A. JorbA, M. Ollé, Invariant curves near Hamiltonian-Hopf bifurcations of $4 D$ symplectic maps, preprint 2003.

[13] A. Jorba, J. Villanueva, Numerical computation of normal forms around some periodic orbits of the RTBP, Physica D 114 (1998), 197-229.

[14] A. Jorba, J. Villanueva, On the normal behaviour of partially elliptic lower dimensional tori of Hamiltonian systems, Nonlinearity 10 (1997), 783-822.

[15] M. OlLÉ, Numerical exploration of bifurcation phenomena associated with complex instability, in Numerical methods for bifurcation problems and Large-Scale dynamical systems, IMA vol. in Maths and its Applications, Springer Verlag, 2000, 319-326.

[16] M. Ollé, J. R. PACHA, The 3D elliptic RTBP: periodic orbits which bifurcate from limiting restricted problems. Complex instability, Astron. Astrophys. 351 (1999), 1149-1164.

[17] M. Ollé, J. R. Pacha, J. Villanueva, Dynamics and bifurcation near the transition from stability to complex instability, in Proceedings of the IV International Symposium HAMSYS-2001. Guanajuato (México), March 19-24, 2001 (to appear).

[18] M. Ollé, J. R. Pacha, J. Villanueva, Dynamics close to a non semisimple 1 : -1 resonant periodic orbit, preprint 2003, web site page http://wwwma1.upc.es/recerca/2003.html.

[19] M. Ollé, D. Pfenniger, Bifurcation at complex instability, in Hamiltonian systems with three or more degrees of freedom, C. Simó (Ed.), NATO Adv. Sci. Inst. Ser. C Math. Phys. Sci. 53, Kluwer Acad. Publ. Dordrecht, Holland, 2000, 518-522.

[20] M. Ollé, D. Pfenniger, Vertical orbital structure and the Lagrangian points in barred galaxies, Astron. Astrophys. 334 (1998), 829-839.

[21] J. R. PACHA, On the quasi-periodic Hamiltonian Andronov-Hopf bifurcation, Ph D. Thesis (2002), UPC, Barcelona, web page site http://tdx.cesca.es.

[22] D. Pfenniger, Numerical study of complex instability. I Mappings, Astron. Astrophys. 150 (1985), 97-111.

[23] D. Pfenniger, Numerical study of complex instability. II Barred galaxy bulges, Astron. Astrophys. 150 (1985), 112-128.

[24] D. Pfenniger, Stability of the Lagrangian points in stellar bars, Astron. Astrophys. 230 (1990), 55-66. 
[25] C. Siegel, J. Moser, Lectures on celestial mechanics, Springer-Verlag, New York, 1971.

[26] C. Simó, Effective computations in celestial mechanics and astrodynamics. In Modern Methods of Analytical Mechanics and Their Applications, V. V. Rumyantsev and A. V. Karapetyan (ed.), vol. 37 of CISM Courses and Lectures, Springer Verlag, 1998.

[27] V. Szebehely, Theory of orbits, Academic Press, New York, 1967.

[28] C.G. Zagouras, Three-dimensional periodic orbits about the triangular equilibrium points of the restricted problem of three bodies, Cel. Mech. 37 (1985), 27-46. 